\title{
PERCEPÇÃO DE RISCOS E CULTURAS DE SEGURANÇA NAS ORGANIZAÇÕES
}

\author{
Maria Luisa Lima
}

\begin{abstract}
Resumo A segurança nas organizações tem constituído recentemente um campo de pesquisa para a qual as ciências comportamentais têm vindo a ser chamadas. Neste artigo, pretende-se (1) salientar a importância dos factores sociais na génese dos acidentes de trabalho (na identificação de uma situação como perigosa, na avaliação da probabilidade de ocorrência de um acidente e na decisão pelo curso de aç̧ão seguro) para mostrar que o chamado erro humano nunca é o erro de um indivíduo isolado, mas o resultado de uma série de processos sociais que culminam num acidente $\mathrm{e}$ (2) mostrar como a forma como é vista a prevenção dos acidentes ao nível das organizaçōes está dependente de uma visăo mais geral da empresa, englobando-se a chamada cultura organizacional de segurança na cultura mais geral da empresa.
\end{abstract}

Palavras-chave segurança nas organizações; acidentes de trabalho; prevençăo; percep̧̧ão de risco.

\section{O problema da segurança nas organizações}

Em Portugal, de acordo com os últimos dados oficiais conhecidos, ocorreram, no ano de 1996, cerca de 220.000 acidentes de trabalho que provocaram directamente a morte a perto de 250 trabalhadores. Embora se possa discutir acerca da credibilidade destes números, eles são consensuais em três aspectos: em primeiro lugar é consensual que estes números não são superiores aos realmente observados; em segundo são consensualmente utilizados como indicadores de segurança nas organizações, e em último são valores elevados que indicam que os acidentes de trabalho são um importante problema nas empresas industriais e de construção. Se bem que a grande maioria dos acidentes de trabalho registados em Portugal não sejam mortais, são os acidentes mortais, os mais visíveis e noticiados que produzem mudanças nas legislações e nas condições de trabalho. Assim, foi depois do acidente de Seveso, em 1976, que nos diversos países da Europa se começaram a dar passos decisivos no sentido da modificação das legislações de segurança nas empresas, embora nem sempre tenha sido igualmente modificada a legislação criminal de modo a punir com mais severidade as suas infracções.

Os estudos que procuram sistematizar as causas dos acidentes de trabalho mostram que os acidentes são produzidos por uma multiplicidade de factores que

Maria Luisa Lima, Instituto Superior de Cincias do Trabalho e da Empresa, Lisboa. 
contribuem para a sua ocorrência num determinado momento e que a esmagadora maioria dos acidentes se deve, directa ou indirectamente, ao chamado erro humano. Este facto tem vindo a trazer as ciências comportamentais para uma questão que anteriormente era vista apenas como técnica ou como legal. Deste modo, no âmbito das ciências sociais, os acidentes de trabalho deram origem a algumas questōes relevantes de investigação, que analisaremos neste artigo.

\section{Acidentes e percepção de risco}

As perspectivas iniciais que tentaram ligar o risco aos acidentes de trabalho considerando o papel dos trabalhadores defendem a ideia de que os comportamentos de insegurança são resultado de uma incorrecta avaliação dos riscos no local de trabalho. Esta posição parte do princípio de que existe a possibilidade de se fazer uma correcta avaliação destes riscos, que é deixada ao cuidado dos técnicos da análise de riscos e, em termos de prevenção dos acidentes, defende a noção de que os trabalhadores devem ser ensinados acerca dos riscos que correm. Esta perspectiva é semelhante à que ocorria no âmbito da comunicação de riscos mais gerais ao público, e que também partia do princípio que os técnicos de análise de risco sabiam calcular os riscos que a população corria, pelo que quando as prioridades dos técnicos e as dos cidadãos não coincidiam, se desqualificava a posição destes como irracional ou ignorante. Os estudos levados a cabo por um grupo de psicólogos investigadores no Eugene Decision Research Group, de Oregon, teve um grande impacte na mudança desta concepção. Baruch Fischhoff, Paul Slovic e colaboradores (1978), ao procurarem definir o que, na perspectiva do público, poderia ser considerado um balanço equilibrado entre os riscos e os benefícios associados às diferentes tecnologias, utilizam e operacionalizam um conceito que até aí era considerado intangível: o conceito de risco percebido. Os resultados que obtiveram apresentaram ainda a vantagem de demonstrar que a definição unidimensional de risco utilizada pelos técnicos (basicamente, a probabilidade de ocorrência de um acontecimento pesado pela sua gravidade) em nada se assemelha à multidimensionalidade que o conceito tem para os cidadãos. De facto, $a$ introdução e operacionalização do conceito de rísco percebido permitiu mostrar que as avaliaçōes de risco feitas pelos leigos são estruturadas e previsíveis, mas que se baseiam em informaçōes e valores diferentes das dos técnicos. O seu carácter multidimensional contrasta com as operacionalizaçōes técnicas de risco, como o número de vítimas a que o risco dá origem ou a sua probabilidade de ocorrência. Slovic (1987) resume da seguinte forma este ponto:

As percepçōes e atitudes dos indivíduos [face ao risco] năo são apenas determinadas por informaçōes como as que aparecem nestas tabelas (risco por hora de exposiçăo ou a probabilidade de morte por ano), mas também por uma variedade de características quantitativas e qualitativas que surgem nas nossas análises. (...) Em resumo, para as pessoas, risco significa mais do que o número de mortes esperadas (p. 285). 
Neste sentido, risco percebido é definido como o "conjunto das crenças, atitudes, avaliações e sentimentos das pessoas acerca das situações de perigo e dos riscos a elas associadas" (Pidgeon et al., 1992).

Pode argumentar-se, no entanto, que os estudos da percepção de riscos para a população em geral não pode ser directamente transposta para o caso da percepção de risco nas organizaçōes, uma vez que no primeiro caso se trata de estudos descontextualizados, onde as pessoas avaliam riscos a que não estão directamente expostas, quando no caso dos riscos organizacionais os trabalhadores estão expostos e contactam diariamente com as situações de perigo. De facto, neste caso pareceria natural encontrarmos um maior consenso entre as avaliaçồes de risco feitas por técnicos e leigos. No entanto, os estudos realizados no âmbito organizacional mostram as mesmas tendências de resultados da avaliaçăo de riscos em geral: situaçōes familiares, com que os trabalhadores contactam mais frequentemente e que são as formas mais comuns de acidentes numa empresa săo subavaliadas, enquanto que as situaçōes menos familiares e mais salientes săo sobre-avaliadas (Hale, 1971; Zimolong, 1985; Lischenstein et al., 1978); a percepção de controlo sobre as situaçōes de ameaça diminui a preocupação com os riscos (Hale, 1987). Estes estudos mostram que a forma como o risco é percebido no contexto de trabalho é, em muitos aspectos, semelhante à forma como ela é pensada em contextos mais gerais. No entanto, os estudos sobre a relação entre risco percebido e acidentes também não mostram a existência de nenhuma relação directa entre os dois.

As perspectivas mais actuais que relacionam a percepção do risco e os acidentes em vez de defenderem uma relação directa entre os dois, postulam uma relação que é mediada por uma série de factores de ordem individual, de ordem social e organizacional. Sistematizaremos esses factores a partir das três etapas usualmente definidas na cadeia de avaliaçōes envolvidas na escolha individual de um comportamento de segurança.

\section{Identificaçāo da situação como perigosa}

A categorização de uma situação como perigosa deverá ser o primeiro passo para a opção por comportamentos de segurança. No entanto, há uma série de factores que influenciam a identificação dos sinais de perigo no ambiente:

- a condição física do trabalhador é uma variável importante. A fadiga, doença, o álcool ou as drogas modificam as condiçōes de percepção e afectam a detecção de sinais de perigo;

- por outro lado, há perigos no ambiente de trabalho que, mesmo em condiçôes de saúde, não são detectáveis. As radiaçōes, a electricidade, determinados gases não săo detectáveis pelos instrumentos sensoriais humanos e precisam de estar assinalados;

- quando existem, os sinais de aviso podem ainda não ser detectados. Isto pode dever-se a um mecanismo de habituação do indivíduo aos estímulos de perigo (perdendo estes o seu valor de sinal) ou mesmo à impossibilidade de 
os percepcionar (por estarem a trabalhar em condiçốes de muito ruido ou de muito fumo, ou por terem colocado auriculares que impedem ouvir uma sirene de aviso);

- mesmo que sejam detectados os sinais de perigo, a importância que lhes é dada depende em grande parte de factores sociais. A mera presença de outros a realizarem um trabalho semelhante leva a que os indivíduos categorizem a situação não perigosa (Latané \& Darley, 1968), e a coesão grupal em grupos em que a norma é a negligência ou o desprezo por regras de segurança modificam a importância dada à identificação dos sinais de aviso (Janis, 1982).

Avaliação da probabilidade de ocorrência de acidente

Após a identificação da situação como perigosa, a opção por um comportamento seguro está dependente de uma avaliação da probabilidade de acidente. Também neste caso há uma série de factores que influenciam a identificação dos sinais de perigo no ambiente:

- vimos que acidentes conhecidos e frequentes são sub-avaliados, enquanto que as situações menos acidentes menos familiares, mais salientes e com imagem mais expressivas são sobre-avaliadas (Hale, 1971; Zimolong, 1985);

- mas a avaliação de probabilidades é uma tarefa complexa, que tendemos a ignorar no nosso comportamento rotinizado. A maioria das aç̧ões que desenvolvemos diariamente, estão mais ligadas a um funcionamento automático guiado por scripts do que por um pensamento sistemático que se baseia na avaliação de probabilidades (Wagenaar, 1992). É por isso possível que, em situaçōes de rotina, esta fase seja mesmo completamente dispensada;

- por outro lado, a avaliação de probabilidade de acontecimentos negativos é sistematicamente enviesada por factores que envolvem implícita ou explicitamente a comparação com outros. Um destes mecanismos funcionalmente adaptativo designado por optimismo irrealista (Weinstein, 1980) consiste numa sistemática subavaliação da probabilidade de ocorrência de uma acidente ao próprio comparativamente com a dos outros. Deste modo, o optimismo cria uma percepção de invulnerabilidade comparativamente aos outros, o que leva a uma subavaliação dos riscos que corre. Fenómenos semelhantes acontecem com outro tipo de ilusões relativos às próprias capacidades (ilusōes de competência) e de controlo em situações de perigo (ilusões de controlo);

- também as normas grupais e a cultura organizacional podem contribuir para uma subavaliação das probabilidades de ocorrência de acontecimentos negativos. Num estudo realizado no sector bancário português (Machado $e$ Lima, 1996) verificámos que em culturas com uma grande componente de apoio os seus membros minimizam a probabilidade de poderem vir a ser vítimas de desemprego, enquanto em culturas de inovação esses riscos são avaliados como mais prováveis. 


\section{Decisão pelo curso de acção correcto}

Nesta perspectiva racionalista, a escolha de um comportamento alternativo ao de risco implica a existência e acessibilidade de alternativas de acção seguras. No entanto a pesquisa nesta área tem mostrado que:

- muitas vezes as organizações dão por garantido que os comportamentos adequados em caso de acidente são bem conhecidos quando de facto eles não o são. A análise dos acidentes tem mostrado que existe frequentemente nas chefias um falso sentido de segurança derivado da crença de que "as regras previnem os acidentes", sem verificarem a divulgação destas regras (Toft e Reynolds, 1994);

- mesmo que saibam os comportamentos adequados, podem duvidar da sua eficácia, podem não acreditar na sua capacidade para os realizar, ou mesmo podem achar que os incómodos não justificam o ganho em termos de segurança (Rogers, 1983).

Conclusão: a importância dos factores sociais e organizacionais nas opções de segurança

Vimos que a maioria dos acidentes de trabalho se devem ao que costuma ser designado erro humano. Esta designação indicia a existência de uma falha num indivíduo, mas vimos que, mesmo quando pode ser este o caso, o seu comportamento pode ser influenciado por variáveis sociais e organizacionais. O modelo de análise de acidentes proposto por Turner (1976, 1978; Pidgeon, 1996) analisa estes acontecimentos num nível de análise claramente organizacional, mostrando que, apesar das melhores intenções de todos os envolvidos, os objectivos de sistemas tecnológicos que funcionam de uma forma segura podem ser subvertidos por processos muito comuns da vida organizacional. Nesta perspectiva, os desastres que acontecem nas organizações não são fruto do acaso, nem são simplesmente falhas tecnológicas, nem podem ser normalmente imputados a um único indivíduo. São processos que se desenrolam no tempo, acumulando-se erros que são mal interpretados durante um período de incubação, aumentando gradualmente a vulnerabilidade do sistema. Por outro lado, em organizações complexas, a informação total sobre um determinado fenómeno não está disponível num único indivíduo ou grupo, mas encontra-se dispersa em diversas estruturas da organização, cada uma delas especializada e atenta a aspectos particulares do todo. Finalmente, a ocorrência de um acontecimento precipitante (um erro humano crítico, um acontecimento exterior ou a operação anormal do sistema) provoca o acidente. Este pode ter a potencialidade de obrigar toda a organização a questionar-se sobre a relação entre os seus pressupostos de segurança e as práticas organizacionais, e deste modo induzir a mudanças futuras. Esta perspectiva sociotécnica analisa o problema da segurança organizacional de uma perspectiva mais alargada, dando um claro peso aos factores sociais na atenuação da percepção de perigo, e no aumento da vulnerabilidade organizacional (e não individual) ao acidente. 


\section{Prevenção de acidentes e produção de normas de segurança}

A intervenção ao nível da segurança nas organizaçōes deve, como vimos, levar em conta tanto as características do ambiente objectivo de trabalho, como das pessoas e as características das organizaçōes. As perspectivas de intervenção sobre a segurança organizacional conceptualizam de forma diferente a produção das regras de segurança e o contributo dos actores organizacionais.

Wagenaar (1992) defende que é impossível ensinar procedimentos de segurança, porque a grande maioria das nossas aç̧ões são controladas automaticamente e não são decisōes conscientes. Nas suas palavras, "as pessoas correm riscos, mas não os assumem" (p. 279). Assim sendo, o funcionamento normal não passa pelas etapas racionais da tomada de decisão, e a tarefa de prevenção de acidentes deve ser executada pelos indivíduos que planeiam e desenham o espaço e o seu funcionamento. Deste modo, as campanhas de segurança deveriam ser dirigidas não aos trabalhadores das empresas mas aos próprios arquitectos $\mathrm{e}$ engenheiros que definem o espaço.

Numa posição radicalmente oposta, Moore (1991) defende que os riscos são mais facilmente identificados e controlados pelos indivíduos que estão nas situaçōes de trabalho, que conhecem as características das suas funçōes e o estado em que estão os equipamentos. Deste modo, defende um sistema de avaliação de riscos de baixo para cima, o que implica a informação e o envolvimento dos trabalhadores no processo de avaliação de riscos, e a existência de um processo de participação na definição das normas de segurança na empresa, com os respectivos canais bidireccionais de comunicação.

$\mathrm{Na}$ linha da perspectiva sociotécnica apresentada atrás, Pidgeon (1996) defende que a segurança organizacional depende da acção concertada a pelo menos quatro níveis: empenhamento da alta direç̧ão relativamente à segurança; partilha da preocupação com perigos; normas e regras de segurança realistas e flexíveis; $\mathrm{e}$ uma contínua reflexão sobre a prática, através de mecanismos de monitorização, análise e feedback.

\section{Culturas organizacionais de segurança}

Nos últimos anos temos começado a ouvir referências na literatura a culturas de segurança nas empresa. Este conceito, começou a ser utilizada nos relatórios acerca do acidente em Chernobyl, e foi imediatamente alvo de um grande interesse empírico ao nível da sua operacionalização, na procura de formas de diagnóstico eficiente de falhas na segurança das empresas. No entanto, os diversos instrumentos produzidos (Brown e Holme, 1986, Dedobbeleer e Beland, 1991) situam-se mais ao nível das atitudes individuais face à segurança e de procedimentos administrativos do que de processos organizacionais mais gerais, perdendo-se pelo caminho o carácter partilhado e social que está implícito no conceito de cultura. Para além disso, as relações entre os resultados destes instrumentos de cultura organizacional e o número de acidentes das empresas onde ocorrem tem sido sistematicamente 
baixas (Mearns e Flin, 1995), o que tem remetido a discussão para a já velha questão em psicologia social entre a relação entre atitudes e comportamentos.

Na nossa perspectiva, a ideia de uma cultura de segurança é simultaneamente muito importante e muito problemática. Em primeiro lugar, ao abordarmos a questão dos acidentes em termos de culturas de segurança estamos necessariamente a sair do âmbito da culpa individual por um acidente, e a referir-nos a um conjunto de forças grupais que actuam ao longo do tempo no seu desencadear. Nesse sentido, a cultura de segurança deveria ser vista como apenas uma dimensão da cultura da empresa, na qual fazem sentido: (1) estratégias mais activas ou mais reactivas de produção e de implementação das regras de segurança, e (2) formas mais ou menos globais de aprendizagem organizacional após os acidentes. Por outro lado, o perigo inerente à ideia de culturas de segurança é o seu potencial retórico, que pode contribuir para o aumento da vulnerabilidade organizacional ao desastre, em vez de potenciar as suas defesas face ao acidente. Nas palavras de Clarke (1993): "As culturas organizacionais podem estar organizadas de modo a aumentar as ideias de risco ou de segurança. Podem também isolar os seus membros de pontos de vista divergentes. $\mathrm{E}$, deste modo, podem perpetuar mitos de controlo e manter a ficção de que o sistema é seguro" (p. 687).

\section{Referências}

Brown, R. L., \& Holmes, H. (1986). The use of a factor analytic procedure for assessing the validity of an employee safety climate model. Accident Analysis and Prevention, $18,455-470$.

Clarke, L. (1993). Drs Pangloss and Strangelove meet organizational theory: High reliability organizations and nuclear weapons accidents. Sociological Forum, 8, 675-689.

Clarke, S. G. (1995, May). Strategic decision making: Implications for risk management. Paper presented to the ESRC Conference on Risk in Organizational Settings, Londres.

Dedobeleer, N., \& Beland, F. (1991). A safety climate measure for construction sites. Journal of Safety Research, 22, 97-103.

Fischhoff, B., Slovic, P., Lichtenstein, S., Read, S., \& Coombs, B. (1978). How safe is safe enough? A psychometric study of attitudes toward technological risks and benefits. Policy Science, 9, 127-152.

Hale, A. (1971). Appreciation of risks at work. In P. Powell, M. Hale, J. Martin, \& M. Simon (Eds.), 2000 Accidents: A shop floor study of their cause based on 42 months' continuous observation (pp. 90-92). Londres: National Institute of Industrial Psychology.

Hale, A. (1987) Subjective risk. In W. T. Singleton, \& J. Hovedon (Eds.), Risk and decisions (pp. 67-85). Chichester: John Wiley.

Janis, 1. L. (1982). Groupthink. Boston, MA: Houghton-Mifflin.

Darley, J. M., \& Latané, B. (1968). Bystander intervention in emergencies: Diffusion of responsability. Journal of Personality and Social Psychology, 8, 377-383. 
Lischenstein, S., Slovic, P., Fischhoff, B., Layman, B., \& Combs, B. (1978). Judged frequency of lethal events. Journal of Experimental Psychology: Human Learning and Memory, 4, 551-578.

Machado, R., \& Lima, M. L. (1996, Novembro). Ameaça de desemprego: Stress e estratégias de coping em diferentes culturas organizacionais. Comunicação apresentada no IV Simpósio de Investigação em Psicologia, Lisboa.

Mearns, K., \& Flin, R. (1995, May). Perception of risk in organizational settings. Paper presented to the ESRC Conference on Risk in Organizational Settings, Londres.

Moore, R. (1991). The price of safety: The market, workers rights and the law. Londres: Institute of Employment Rights.

Pidgeon, N. F. (1996). The limits to safety? Culture, learning and man-made disasters. The Journal of Contingencies and Crisis Management.

Pidgeon, N. F., Hood, C., Jones, D., \& Turner, B. A. (1992). Risk perception. In Risk: analysis, perception and management (pp. 21-43). Londres: The Royal Society.

Rogers, R. W. (1983). Cognitive and physiological processes in attitude change: A revised theory of protection motivation. In J. Caccioppo, \& R. Petty (Eds.), Social Psychophisiology (pp. 153-176). Nova Iorque: Guilford Press.

Slovic, P. (1987). Perception of risk. Science, 236, 280-285.

Toft, B., \& Reynolds, S. (1994). Learning from disasters: A management approach. Oxford: Butterworth-Heinemann.

Turner, B. A. (1978). Man-made disasters. Londres: Wykeham Science Press.

Wagenaar, W. A. (1992) Risk taking and accident causation. In J. F. Yates (Ed.), Risk-taking behaviour (pp. 257-281). Chichester: John Wiley.

Weinstein, N. D. (1980). Unrealistic optimism about future life events. Journal of Personality and Social Psychology, 39, 806-820.

Zimolong, B. (1985). Hazard perception and risk estimation in accident causation. In R. E. Eberts, \& C. G. Eberts (Eds.), Trends in Ergonomics/Human Factors II (pp. 463-470). Amesterdão: Elsevier Science Publishers.

Zohar, D. (1980). Safety climate in industrial organizations: theoretical and applied implications. Journal of Applied Psychology, 65, 96-102.

Risk Perception and Safety Cultures in Organisations (abstract) Organisational safety is a recent field of research in behavioural sciences. In this paper we aim at (1) stressing the importance of social factors on the origin of work accidents (on the classification of a situation as dangerous, on the judgement of probabilities associated with accidents, and on the individual decision for a safe behaviour). This point supports our claim that accidents cannot be conceived as the error of a single individual, but as the result of ongoing social processes. (2) showing that the organisational strategies for accident prevention are dependent on a more global view of the organisation. Specifically, we maintain that safety culture should be conceived as a part of a more global organisational culture. 